\title{
Reparación de Lesiones Musculares por Incisión Quirúrgica Coadyuvada con una Formulación Basada en Miel Nativa (Ulmoplus®). Estudio Experimental en Modelo Animal de Conejo (Oryctolagus cuniculus)
}

\author{
Repair of Muscle Injuries by Coadjugated Surgical Incision with a Native Honey Formulation \\ (Ulmoplus( $\left.{ }^{\circledR}\right)$. Experimental Study on Rabbit Animal Model (Oryctolagus cuniculus)
}

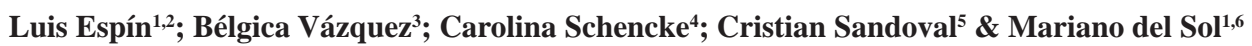

ESPÍN, L.; VÁSQUEZ, B.; SCHENCKE, C.; SANDOVAL, C. \& DEL SOL, M. Reparación de lesiones musculares por incisión quirúrgica coadyuvada con una formulación basada en miel nativa (Ulmoplus®). Estudio experimental en modelo animal de conejo (Oryctolagus cuniculus). Int. J. Morphol., 38(2):492-498, 2020.

RESUMEN: Para promover la reparación y regeneración muscular, se han desarrollado diferentes estrategias, sin embargo, aún no existe un tratamiento único aceptado. Investigaciones relacionan los efectos terapéuticos de la miel en el proceso de cicatrización de heridas de diversas etiologías. Considerando lo anterior, el propósito de este estudio fue evaluar los efectos de una formulación basada en miel nativa (Ulmoplus ${ }^{\circledR}$ ) en la reparación de lesiones musculares por incisión quirúrgica. A cinco conejos (Oryctolagus cuniculus) se les realizó una incisión de $20 \mathrm{~mm}$ de largo y $3 \mathrm{~mm}$ de ancho en la región anterior de cada pierna, atravesando la epidermis, dermis y el músculo tibial anterior, hasta alcanzar el periostio de la tibia. La pierna izquierda de cada animal fue clasificada como grupo sutura (S) y la derecha como grupo sutura más Ulmoplus ${ }^{\circledR}(\mathrm{S}+\mathrm{U})$. Posteriormente, al grupo $\mathrm{S}+\mathrm{U}$ se le aplicó Ulmoplus ${ }^{\circledR}$ en toda la extensión de la herida. Luego, en ambos grupos, la incisión fue suturada con cuatro puntos intradérmicos (vicryl 2/0). Un animal fue considerado como control para obtener imágenes histológicas del músculo tibial anterior sin injuria. A los 10 días post incisión, fueron tomadas las biopsias del músculo y posteriormente fueron procesadas para su observación en microscopio de luz. El estudio se llevó a cabo considerando las recomendaciones de la Guía para el Cuidado y Uso de Animales de Laboratorio. A los 10 días post incisión, las injurias, en ambos grupos de animales, se encontraban cerradas y sin signos de infección. El músculo tibial anterior de los especímenes del grupo S se encontraba en la fase de reparación, con procesos concomitantes de fagocitosis del tejido necrotizado, regeneración de miofibras y formación de tejido conectivo cicatrizal. El músculo tibial anterior de los animales del grupo $\mathrm{S}+\mathrm{U}$ se encontraba en la fase de remodelación, ya que la arquitectura muscular se evidenciaba restablecida. Ulmoplus ${ }^{\circledR}$, como coadyuvante en el proceso de cierre de heridas quirúrgicas de músculo esquelético utilizando sutura convencional, es una buena alternativa, ya que acelera el proceso de reparación muscular, y reduce el tejido conectivo cicatrizal.

PALABRAS CLAVE: Conejo; Músculo; Incisión; Reparación; Sutura; Ulmoplus®.

\section{INTRODUCCIÓN}

Se sabe que el músculo esquelético tiene la capacidad de regenerar nuevas fibras musculares después de haber sido dañado por una lesión, o como consecuencia de enfermedades (Huard et al., 2002). El proceso de reparación muscular parece ser similar en los diferentes mamíferos y, en rasgos generales, sigue el mismo programa para cada músculo. En circunstancias normales, el músculo esquelético adulto de mamíferos es un tejido estable con muy poca rotación de núcleos, sin embargo, después de una lesión, el músculo esquelético tiene la notable capacidad de iniciar un proceso de reparación rápido y extenso que previene la pérdida de masa muscular (Fu et al., 2018).

Independientemente del tipo o la gravedad de la lesión, la reparación del músculo esquelético sigue un patrón establecido, que se puede dividir en tres fases: la fase de

\footnotetext{
${ }^{1}$ Programa de Doctorado en Ciencias Morfológicas, Facultad de Medicina, Universidad de La Frontera, Temuco, Chile.

${ }^{2}$ Universidad Central del Ecuador, Quito, Ecuador.

${ }^{3}$ Universidad de Tarapacá, Arica, Chile.

${ }^{4}$ Universidad Autónoma de Chile, Temuco, Chile.

${ }^{5}$ Departamento de Ciencias Preclínicas, Facultad de Medicina, Universidad de La Frontera, Temuco, Chile.

${ }^{6}$ Centro de Excelencia en Estudios Morfológicos y Quirúrgicos (CEMyQ), Universidad de La Frontera, Temuco, Chile.
} 
ESPÍN, L.; VÁSQUEZ, B.; SCHENCKE, C.; SANDOVAL, C. \& DEL SOL, M. Reparación de lesiones musculares por incisión quirúrgica coadyuvada con una formulación basada en miel nativa (Ulmoplus ${ }^{\circledR}$ ). Estudio experimental en modelo animal de conejo (Oryctolagus cuniculus). Int. J. Morphol., 38(2):492-498, 2020.

destrucción/inflamación, de reparación y de remodelación (Lehto \& Järvinen, 1991; Huard et al.; Järvinen et al., 2005). La fase inicial de la reparación muscular se caracteriza por necrosis del tejido dañado y activación de la respuesta inflamatoria. Esta fase es seguida rápidamente por la activación de células miogénicas para proliferar, diferenciarse y fusionarse, lo que conduce a la formación de miofibras y reconstitución de un aparato contráctil funcional (Charge \& Rudnicki, 2004). Por lo tanto, el episodio inicial en este proceso es la lesión de la membrana, donde los mecanismos de necrosis son similares e independientes de la causa. Para minimizar la necrosis, la primera reacción de la célula es separar las porciones muertas de las recuperables, lo que hace por generación de nueva membrana, cubriendo las regiones todavía recuperables. Eso ocurre rápidamente y es evidente en las primeras horas. Siguiendo un programa preexistente coordinado por la expresión o inhibición de una serie de péptidos, continúan las secuencias de necrosis, inflamación, fagocitosis, revascularización, creación de nuevos miocitos y reinervación (Chambers \& McDermott, 1996).

Aunque las dos primeras fases del proceso de reparación muscular son similares entre los diferentes tipos de músculos, independiente de la causa de la lesión, la cinética y amplitud de cada fase pueden variar según la extensión de la lesión, el músculo lesionado o el modelo animal (Mitchell et al., 1992). Sin embargo, el factor primordial para la recuperación de las fibras es la presencia de una membrana basal intacta y la sincronización que involucra la activación de varias respuestas celulares, entre las que se destacan las células satélites (Järvinen et al., 2000; Järvinen et al., 2005; Forcina et al., 2019). Para el proceso de regeneración, la activación de células satélite musculares es un elemento clave, donde se han identificado factores de señalización, pero sus funciones aún no se han definido por completo (Chen \& Shan, 2019). Además, la evidencia reciente respalda la posible contribución de las células madre en el proceso de regeneración muscular. En particular, las células madre derivadas de la médula ósea y de los músculos contribuyen a la nueva formación de miofibras y al conjunto de células satélite después de la lesión (Klimzak et al., 2018).

El músculo esquelético puede regenerarse completa y espontáneamente en respuesta a lesiones menores, como la tensión. En contraste, después de lesiones graves, la curación muscular es incompleta, lo que a menudo resulta en la formación de tejido fibrótico que deteriora la función muscular (Laumonier \& Menetrey, 2016). El músculo esquelético representa hasta el $40 \%$ de la masa corporal total siendo sus lesiones muy comunes en la práctica clínica (Mori et al., 2015), en atletas y otros ámbitos, como en accidentes automovilísticos, laborales o en secuelas de procedimientos quirúrgicos, entre otros (Sánchez \& Segovia, 2017). Para promover la reparación y regeneración muscular, se han desarrollado diferentes estrategias en el siglo pasado y especialmente durante las últimas décadas, que incluyen técnicas quirúrgicas, fisioterapia, biomateriales e ingeniería de tejido muscular, así como terapia celular (Pereira et al., 2013). Aunque los investigadores han estudiado ampliamente varios enfoques para mejorar la reparación muscular, su tratamiento actualmente es controversial. Por lo tanto, existe una gran necesidad de desarrollar nuevos métodos y materiales que promuevan la reparación del músculo esquelético y la regeneración funcional.

Considerando este contexto, varios estudios validan la acción de la miel en la cicatrización (Gethin \& Cowman, 2005; Ranzato et al., 2013; Majtan, 2014), centrándose en sus propiedades biológicas y físicas para el tratamiento de heridas de diversas etiologías, reavivando el interés en la atención clínica (Seckam \& Cooper, 2013; Vandamme et al., 2013). Aunque la miel se produce en todo el mundo, su potencial biológico debe evaluarse según el origen. En Chile se producen varios tipos de miel nativa con importantes propiedades biológicas, heredadas según el tipo de fuente floral. La miel del Sur de Chile contiene un gran porcentaje de ulmo (Eucryphia cordifolia), un árbol nativo, que ha demostrado tener propiedades bactericidas, fungicidas, antioxidantes y curativas (Sherlock et al., 2010). Para desarrollar una miel terapéutica para uso clínico, esta miel nativa ha sido suplementada con antioxidantes, lo que culminó con la formulación Ulmoplus ${ }^{\circledR}$ (CORFO I + D-13IDL223290) (Schencke et al., 2018).

Basado en lo anteriormente descrito, el propósito de este estudio fue evaluar los efectos de una formulación basada en miel nativa (Ulmoplus®) en la reparación de lesiones musculares por incisión quirúrgica.

\section{MATERIAL Y MÉTODO}

Tratamiento. Para el tratamiento se utilizó la siguiente formulación: miel monofloral nativa del Sur de Chile con el sello NHF (factor de miel nativa), que certifica niveles significativos de actividad antibacteriana (Fredes et al., 2013), complementada con antioxidantes. Esta formulación fue desarrollada con el apoyo de Corporación para la Promoción de la Producción del Estado de Chile, CORFO (13IDL223290) y protegida bajo el Secreto Comercial (RS N³654), por lo que no puede ser revelada, y se mantiene bajo la licencia Ulmoplus ${ }^{\circledR}$.

Animales. Fueron utilizados seis conejos adultos sanos 
ESPÍN, L.; VÁSQUEZ, B.; SCHENCKE, C.; SANDOVAL, C. \& DEL SOL, M. Reparación de lesiones musculares por incisión quirúrgica coadyuvada con una formulación basada en miel nativa (Ulmoplus®). Estudio experimental en modelo animal de conejo (Oryctolagus cuniculus). Int. J. Morphol., 38(2):492-498, 2020.

(Oryctolagus cuniculus) de 4,3 kg aproximadamente, provenientes del Centro de Excelencia en Estudios Morfológicos y Quirúrgicos (CEMyQ) de la Universidad de La Frontera, Temuco, Chile. Fueron alimentados con pellet y agua $\mathrm{ad}$ libitum y mantenidos en condiciones ambientales controladas respecto a temperatura, ruido ambiental y ciclos de 12 horas luz / 12 horas oscuridad. El estudio se llevó a cabo de acuerdo con el protocolo para la supervisión diaria de animales, de la Guía de Aspectos Bioéticos de Experimentos con Animales (Wolfensohn \& Lloyd, 2013), y las directrices establecidas en la Guía para el Cuidado y Uso de Animales de Laboratorio (2011).

Modelo de injuria. A cinco animales se les realizó una incisión de $20 \mathrm{~mm}$ de largo y $3 \mathrm{~mm}$ de ancho en la región anterior de cada pierna, atravesando la epidermis, dermis y el músculo tibial anterior (MTA), hasta alcanzar el periostio de la tibia. La pierna izquierda de cada animal fue clasificada como grupo sutura $(\mathrm{S})$ y la derecha como grupo sutura más Ulmoplus ${ }^{\circledR}(\mathrm{S}+\mathrm{U})$. Posteriormente, al grupo $\mathrm{S}+\mathrm{U}$ se le aplicó Ulmoplus® en toda la extensión de la herida (desde la epidermis hasta el periostio). Luego, en ambos grupos, la incisión fue suturada con cuatro puntos intradérmico de ácido poliglicólico (vicryl 2/0). El procedimiento se realizó bajo efectos anestésicos general con una triada anestésica en base a acepromazina $5 \mathrm{mg}$, ketamina $40 \mathrm{mg} / \mathrm{kg}$ y xilazina $5 \mathrm{mg} /$ $\mathrm{kg}$ por vía intramuscular. A los 10 días post incisión fueron tomadas las biopsias abarcando la piel y MTA. Un animal fue considerado como control para obtener imágenes histológicas del MTA sin injuria. Una vez obtenidas las muestras fueron lavadas con solución salina y fijadas en formalina tamponada al $10 \%$ durante 72 horas. Posteriormente, fueron deshidratadas e incluidas en paraplast (Histotec-Merck®). Se realizaron cortes seriados de $5 \mu \mathrm{m}$ de espesor, los cuales fueron montados en portaobjetos y teñidos con Hematoxilina Eosina (HE) y Van Gieson para el análisis histológico y con Sirius Red para el análisis de colágeno tipos I y III. Las láminas histológicas fueron visualizadas bajo microscopio de luz Leica ${ }^{\circledR}$ DM2000 LED y fotografiada con cámara digital Leica ${ }^{\circledR}$ MC170 HD. Para la visualización de las fibras colágenas se utilizó un microscopio Leica ${ }^{\circledR}$ DM750 bajo luz polarizada, con cámara digital Leica® ICC50 HD.

\section{RESULTADOS}

Los conejos respondieron favorablemente a la intervención quirúrgica y el protocolo de control y seguimiento no reportó inconvenientes (Fig. 1A y C). A los 10 días post incisión, las injurias, en ambos grupos, se encontraban cerradas y sin signos de infección (Fig. 1B y D). En el grupo S se observó mayor longitud de la cicatriz y en algunos casos enrojecimiento e inflamación. El grupo $\mathrm{S}+\mathrm{U}$ presentó una cicatriz de menor longitud, más plana y con mejores resultados estéticos.
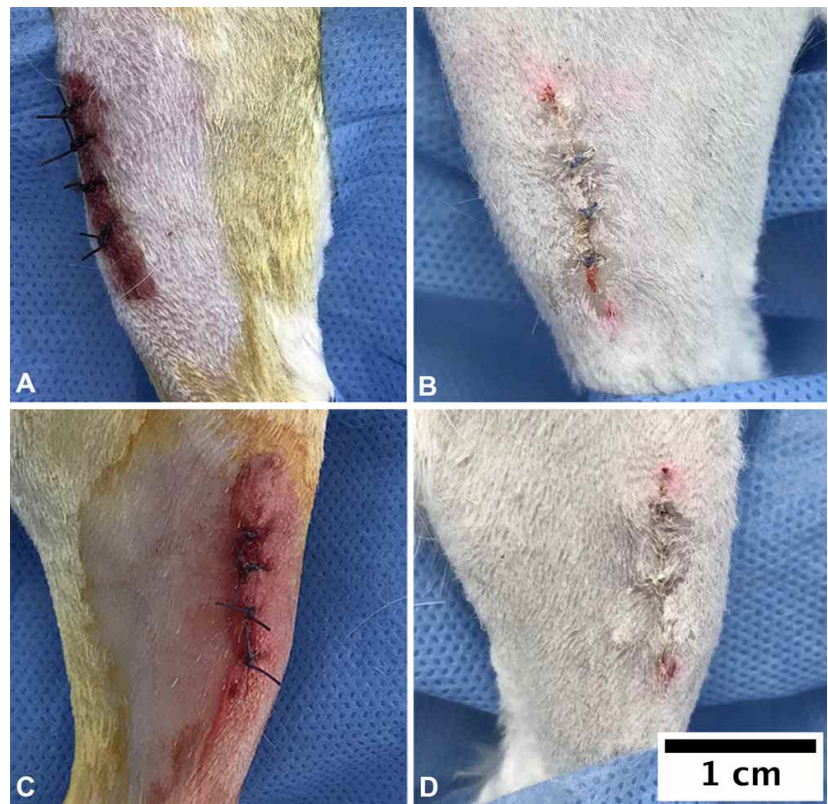

Fig. 1. Región anterior de la pierna de conejo (Oryctolagus cuniculus). A. Grupo sutura (S), día cero. B. Grupo sutura (S), día 10 post incisión. C. Grupo sutura más Ulmoplus ${ }^{\circledR}(\mathrm{S}+\mathrm{U})$, día cero. D. Grupo sutura más Ulmoplus ${ }^{\circledR}(\mathrm{S}+\mathrm{U})$, día 10 post incisión.

Las tinciones con HE obtenidas 10 días posterior a la incisión, mostró que el MTA de los animales del grupo $\mathrm{S}$ se encontraba en la fase de reparación, ya que no se evidenció edema o infiltrado de células inflamatorias (Fig. 2A). En la zona de reparación, se observaron procesos concomitantes de fagocitosis del tejido necrotizado, regeneración de miofibras y formación de tejido conectivo cicatrizal (Fig. 2B).

El comienzo del proceso de regeneración lo demostró la existencia de mioblastos dentro de los cilindros de la lámina basal de las fibras necróticas (Fig. 3A) y la presencia de células miogénicas fusionadas en la zona de la injuria (Fig. 3B). La continuidad del proceso se evidenció con la presencia de miocitos multinucleados cuyas miofibrillas recién formadas mostraban mayor tamaño y mionúcleos de ubicación periférica en la fibra muscular (Fig. 3C). La apariencia de fibras musculares en regeneración y reparación muscular activa también se evidenció por la presencia de fibras con núcleos de ubicación central (Fig. 3D).

El tejido conectivo cicatrizal producido en el sitio de la lesión se caracterizó por presentar fibroblastos, abundante colágeno tipo I, vasos sanguíneos y nervios intramusculares (Fig. 4). 


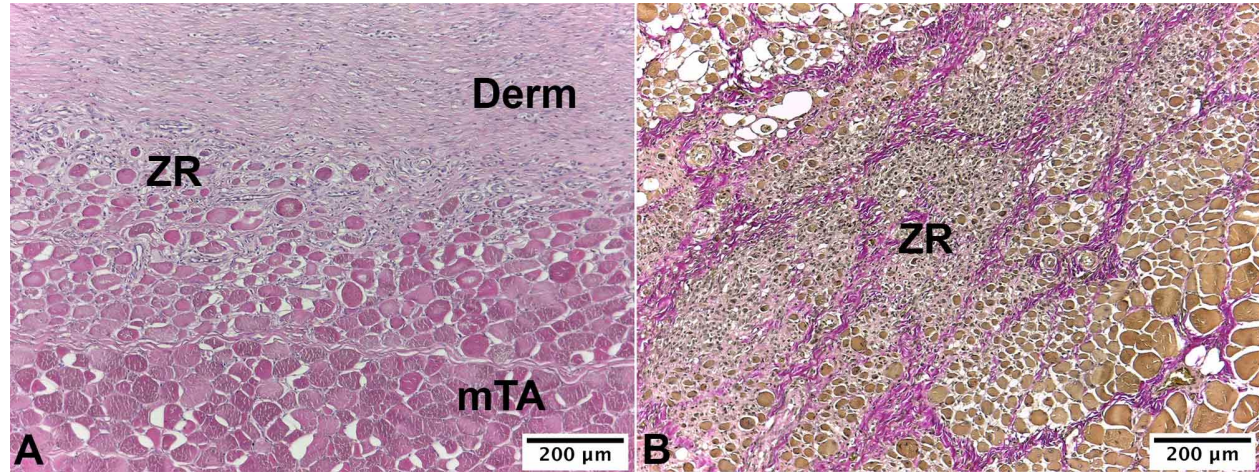

Fig. 2. Biopsia de músculo tibial anterior de conejo (Oryctolagus cuniculus) (grupo S). A. Se observa la dermis (Derm) con abundante cantidad de fibroblastos y fibras colágenas. En la zona de reparación (ZR) del músculo tibial anterior (mTA) se observan miocitos de pequeño calibre recién formadas. B. En la ZR se evidencia abundante tejido conectivo cicatrizal; destacan las fibras de colágeno en color fucsia. Tinciones HE y Van Gieson.

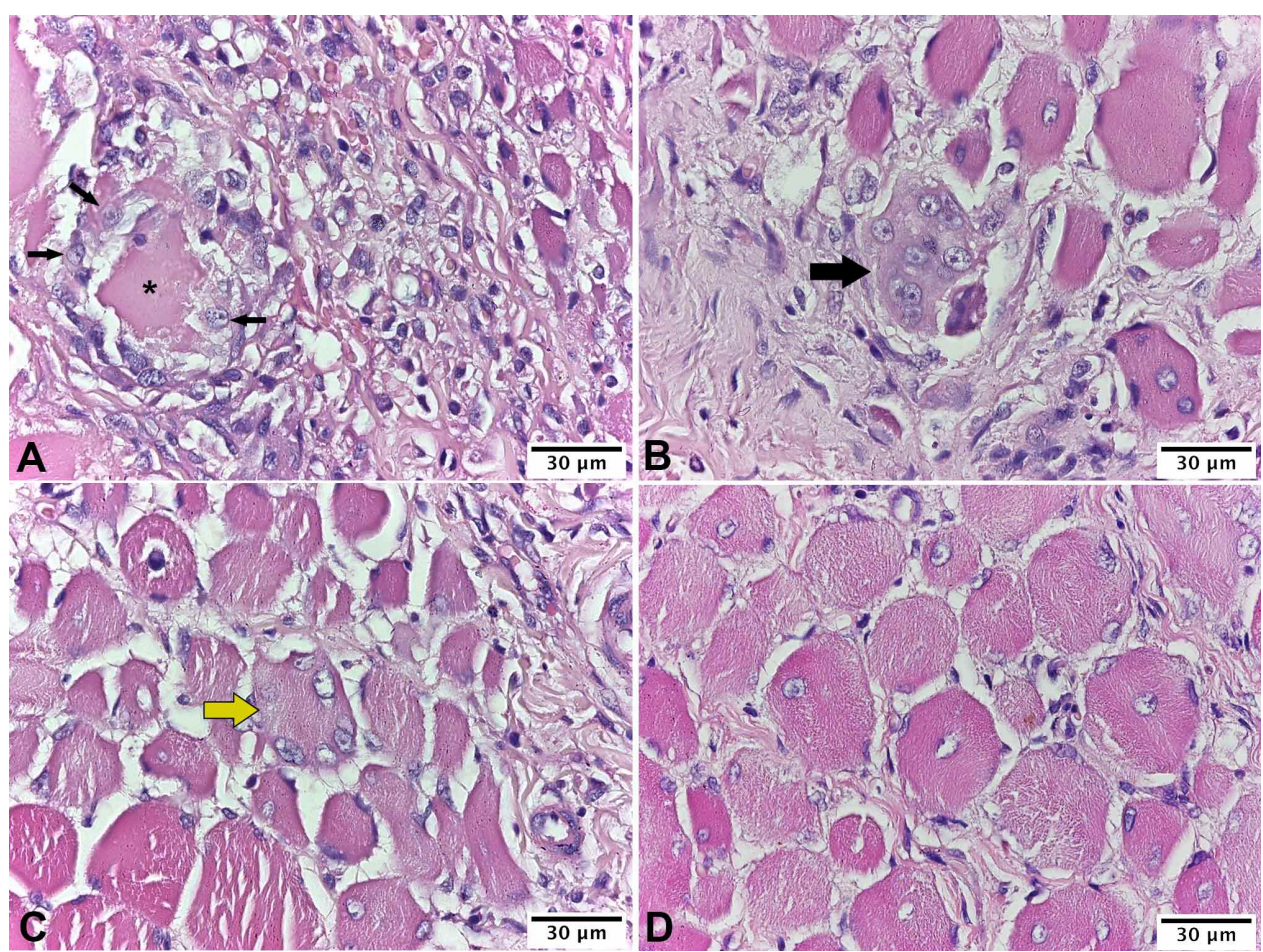

Fig. 3. Biopsia de músculo tibial anterior de conejo (Oryctolagus cuniculus) (grupo S). A. Fibra muscular necrótica $(*)$ rodeada por mioblastos (flecha delgada). B. Sincitio de células miogénicas con múltiples núcleos vesiculares con prominentes nucléolos (fechas gruesa). C. Miocitos con núcleos periféricos (fecha amarilla). D. Miocitos de tamaño y forma variable, algunos presentan núcleos de ubicación centra. Tinción HE.

El MTA de los individuos del grupo S+U se encontraba en la fase de remodelación, ya que la arquitectura muscular se evidenciaba restablecida. En esta fase, la cantidad de tejido conectivo, y en particular el colágeno tipo I, fue menor al observado en el grupo $\mathrm{S}$, sin embargo, parece ser mayor que en el músculo no lesionado. En el grupo $\mathrm{S}+\mathrm{U}$ aún fue posible observar miocitos recién formadas, con núcleos de ubicación central, generalmente más pequeñas, de tamaño variable y con formas redondeadas (Fig. 5).

\section{DISCUSIÓN}

Las lesiones del músculo esquelético son muy frecuentes y plantean problemas desafiantes en relación a su reparación y rehabilitación. A pesar de su importancia clínica, las estrategias óptimas para tratar estas lesiones no están bien definidas. En este sentido, nuestro estudio ha permitido conocer la idoneidad del uso de la miel en la reparación y regeneración de MTA de conejo lesionado por incisión quirúrgica a través de la evaluación de las características histológicas que presentó cada grupo en relación al proceso de reparación muscular.

La lesión muscular siempre será una lesión profunda, en el sentido que compromete desde la piel, fascias superficial y profunda y los elementos que se encuentran en ellas y, por su puesto, el tejido muscular. A los 10 días post incisión, las injurias en ambos grupos se encontraban cerradas evidenciando un proceso de reparación activo, lo que demuestra la excelente capacidad de reparación y regeneración que tiene el tejido muscular, característica ya señalada, entre otros, por Fu et al. No obstante lo señalado, la injuria en la región anterior de la pierna de los animales del grupo $\mathrm{S}+\mathrm{U}$, no solo presentó una cicatriz más estética, sino que también, la reparación del músculo se encontró en una fase más avanzada.

Lo anterior, se basó en lo observado histológicamente en el MTA de ambos grupos. El grupo $S$ presentó en la zona de reparación estructuras que se observan comúnmente 


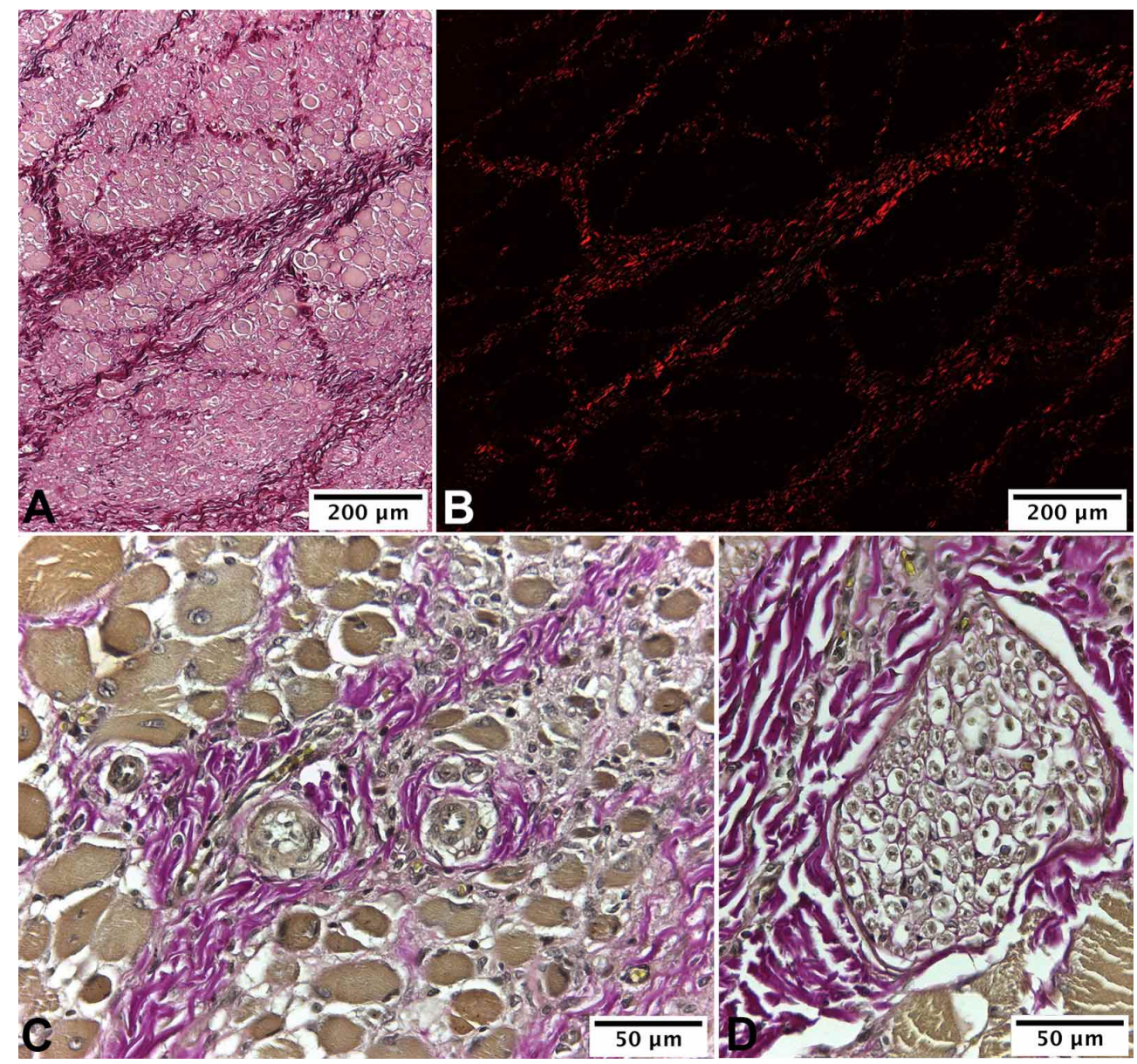

Fig. 4. Biopsia de músculo tibial anterior de conejo (Oryctolagus cuniculus) (grupo S). A y B. En la ZR el tejido conectivo cicatrizal se caracteriza por presentar principalmente colágeno tipo I (color rojo). C. Se observan vasos sanguíneos de distintos calibres. D. Nervios intramusculares. Tinciones Van Gieson y Sirius Red.

durante la fase de reparación, tales como, mioblastos próximos a miocitos necróticos, sincitio de células miogénicas distribuidos en la zona de reparación y miocitos de tamaño variable con núcleos centrales (Järvinen et al., 2005; Turner \& Badylak, 2012). El grupo S+U, en cambio, no mostró una zona de reparación evidente a nivel muscular, ya que la arquitectura del tejido se encontraba restablecida, evidenciando una fase de remodelación; su identificación se basó principalmente en la observación de miocitos de menor diámetro, en los cuales, algunos de ellos presentaban núcleos centrales.

La utilización de las tinciones Van Gieson y Sirius Red, permitió evaluar la presencia de tejido conectivo cicatrizal de mejor manera en la zona de reparación muscular. Nuestros resultados mostraron que el grupo $S$ presentó mayor cantidad de tejido conectivo en comparación al grupo $\mathrm{S}+\mathrm{U}$. Sin embargo, se debe tener en consideración que no es posible determinar si el grupo $\mathrm{S}$ desarrollará fibrosis, debido a que la observación se realizó solo en un determinado momento, el día 10 post injuria, y el proceso de fibrosis comienza aproximadamente durante la segunda semana tras la lesión y no es valorable hasta la cuarta semana (Aurora et al., 2016).
Otro aspecto que destacamos en ambos grupos, es la presencia de vasos sanguíneos y fibras nerviosas intramusculares en el tejido conectivo cicatrizal, ya que para una buena reparación y regeneración muscular es relevante el restablecimiento de la vascularización y la inervación (Turner \& Badylak). Bajo este contexto, estudios demuestran que la miel tiene la capacidad de estimular la angiogénesis en el proceso de curación de heridas, ya que la alta presión osmótica y el peróxido de hidrógeno contenido en niveles bajos en la miel, estimula el desarrollo de nuevos capilares (Molan, 2002; Nakajima et al., 2013).

Lo precedente, podría explicar, no solo la adecuada vascularización del grupo $\mathrm{S}+\mathrm{U}$, sino que además, una aceleración del proceso de reparación muscular, ya que las propiedades cicatrizantes de la miel le permiten promover además, la granulación en la zona de la injuria, estimular linfocitos, fagocitos y fibroblastos y, modular la síntesis de colágeno y su maduración (Schencke et al., 2016).

En base a nuestros resultados concluimos que Ulmoplus ${ }^{\circledR}$, como coadyuvante en el proceso de cierre de 
ESPÍN, L.; VÁSQUEZ, B.; SCHENCKE, C.; SANDOVAL, C. \& DEL SOL, M. Reparación de lesiones musculares por incisión quirúrgica coadyuvada con una formulación basada en miel nativa (Ulmoplus(). Estudio experimental en modelo animal de conejo (Oryctolagus cuniculus). Int. J. Morphol., 38(2):492-498, 2020.

heridas quirúrgicas de músculo esquelético utilizando sutura convencional, es una buena alternativa terapéutica, ya que acelera el proceso de reparación muscular y reduce el tejido conectivo cicatrizal.
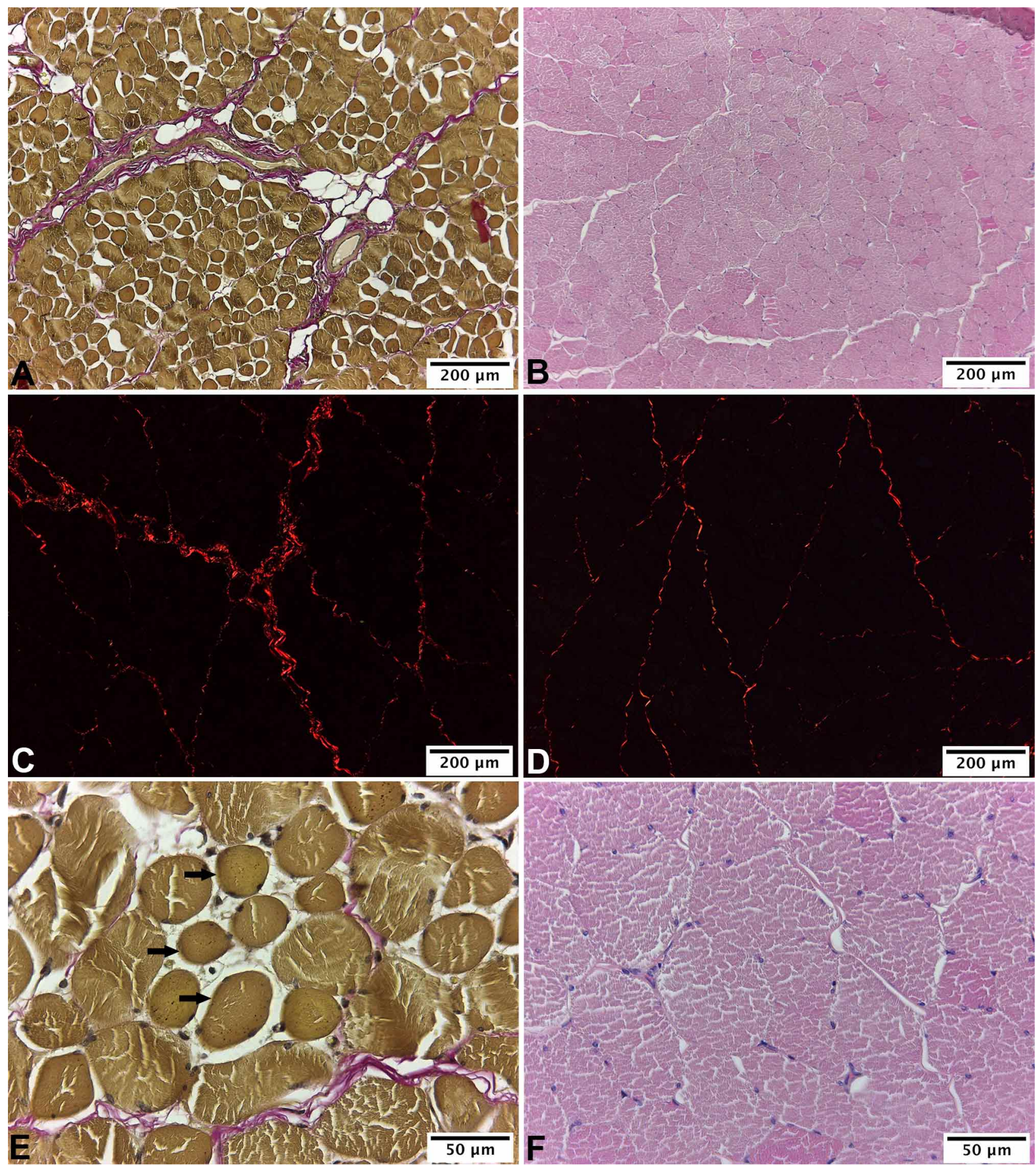

Fig. 5. Biopsia de músculo tibial anterior de conejo (Oryctolagus cuniculus). A, C y D imágenes del grupo S+U. B, D y F imágenes del animal control. En el músculo tibial anterior de los animales del grupo $\mathrm{S}+\mathrm{U}$ se observa mayor cantidad de tejido conectivo, rico en colágeno tipo I (A y C) en comparación con el músculo tibial anterior del animal control (B y D). En el grupo S+U se observan miofibras recién formadas con núcleos periféricos (flechas) (E), mientras que en el animal control solo se observan miofibras maduras (F). Tinciones HE, Van Gieson y Sirius Red. 
ESPÍN, L.; VÁSQUEZ, B.; SCHENCKE, C.; SANDOVAL, C. \& DEL SOL, M. Reparación de lesiones musculares por incisión quirúrgica coadyuvada con una formulación basada en miel nativa (Ulmoplus( ). Estudio experimental en modelo animal de conejo (Oryctolagus cuniculus). Int. J. Morphol., 38(2):492-498, 2020.

ESPÍN, L.; VÁSQUEZ, B.; SCHENCKE, C.; SANDOVAL, C.\& DEL SOL, M. Repair of muscle injuries by coadjugated surgical incision with a native honey formulation (Ulmoplusß). Experimental study on rabbit animal model (Oryctolagus cuniculus) . Int. J. Morphol., 38(2):492-498, 2020.

SUMMARY: To promote muscle repair and regeneration, different strategies have been developed, however, there is still no single accepted treatment. Research relates the therapeutic effects of honey in the wound healing process of various etiologies. The aim of this study was to evaluate the effects of native honey formulation (Ulmoplus ${ }^{\circledR}$ ) in the repair of muscle injuries by surgical incision. In five rabbits (Oryctolagus cuniculus) were made an incision $20 \mathrm{~mm}$ long and $3 \mathrm{~mm}$ wide in the anterior region of each leg, crossing the epidermis, dermis and anterior tibial muscle, until reaching the tibia periosteum. The left leg of each animal was classified as a suture group (S) and the right leg as a suture group plus Ulmoplus ${ }^{\circledR}(\mathrm{S}+\mathrm{U})$. Subsequently, Ulmoplus ${ }^{\circledR}$ was applied to the entire $S+U$ group throughout the entire length of the wound. Then, in both groups, the incision was sutured with four intradermal points (vicryl 2/0). An animal was considered as a control to obtain histological images of the anterior tibial muscle without injury. At 10 days post incision, muscle biopsies were taken and subsequently processed for observation under light microscopy. The study was carried out considering the recommendations of the Guide for the Care and Use of Laboratory Animals. At 10 days post incision, the injuries, in both groups of animals, were closed and without signs of infection. The anterior tibial muscle of the $S$ group specimens was in the repair phase, with concomitant processes of phagocytosis of the necrotized tissue, myofiber regeneration and formation of scar connective tissue. The anterior tibial muscle of the animals of the $\mathrm{S}+\mathrm{U}$ group was in the remodeling phase, since the muscular architecture was evidenced restored. Ulmoplus ${ }^{\circledR}$, as an adjunct in the process of closing surgical wounds of skeletal muscle using conventional suture, is a good alternative, since it accelerates the muscle repair process and reduces scar connective tissue.

KEY WORDS: Rabbit; Muscle; Incision; Repair; Suture; Ulmoplus®.

\section{REFERENCIAS BIBLIOGRÁFICAS}

Aurora A, Corona BT, Walters TJ. A Porcine Urinary Bladder Matrix Does Not Recapitulate the Spatiotemporal Macrophage Response of Muscle Regeneration after Volumetric Muscle Loss Injury. Cells Tissues Organs, 202(3-4):189-201, 2016.

Charge, S. B. \& Rudnicki, M. A. Cellular and molecular regulation of muscle regeneration. Physiol. Rev., 84(1):209-38, 2004.

Chambers, R. L. \& McDermott, J. C. Molecular basis of skeletal muscle regeneration. Can J. Appl. Physiol., 21(3):155-84, 1996.

Chen, B. \& Shan, T. The role of satellite and other functional cell types in muscle repair and regeneration. J. Muscle Res. Cell Motil., 4O(1):1-8, 2019.

Committee for the Update of the Guide for the Care and Use of Laboratory Animals, Institute for Laboratory Animal Research (ILAR), Division on Earth and Life Studies (DELS), National Research Council. Guide for the Care and Use of Laboratory animals, 8th edn. Washington (DC): The National Academies Press, 2011.

Forcina, L.; Miano, C.; Pelosi, L.; Musarò, A. An Overview about the Biology of Skeletal Muscle Satellite Cells Curr. Genomics, 20(1):24-37, 2019.

Fredes, C.; Montenegro, G.; Santander, F.; Jara, C. \& Nuñez, G. Actividad antioxidante y antimicrobiana de mieles monoflorales de plantas nativas chilenas. Bol. latinoam. Caribe plantas med. aromát., 12(13):257-68, 2013.

Fu, X.; Zhao, A. \& Hu, T. Dedifferentiation and musculoskeletal repair and regeneration. In cellular dedifferentiation and regenerative medicine. Springer, Berlin, Heidelberg, 2018. pp. 91-116.
Gethin, G. \& Cowman, S. Case series of use of Manuka honey in leg ulceration. Int. Wound J., 2(1):10-5, 2005.

Huard, J.; Li, Y. \& Fu, F. H. Muscle injuries and repair: current trends in research. $J$. Bone Joint Surg. Am., 84(5):822-32, 2002.

Järvinen, T. A.; Kääriäinen, M.; Järvinen, M. \& Kalimo, H. Muscle strain injuries Curr. Opin Rheumatol., 12(2):155-61, 2000.

Järvinen, T. A.; Järvinen, T. L.; Kääriäinen, M.; Kalimo, H. \& Järvinen, M. Muscle injuries: biology and treatment. Am. J. Sports Med., 33(5):745-64, 2005.

Klimczak, A.; Kozlowska, U. \& Kurpisz, M. Muscle Stem/Progenitor Cells and Mesenchymal Stem Cells of Bone Marrow Origin for Skeletal Muscle Regeneration in Muscular Dystrophies. Arch. Immunol. Ther. Exp. (Warsz), 66(5):341-54, 2018

Laumonier, T. \& Menetrey, J. Muscle injuries and strategies for improving their repair. J. Exp. Orthop., 3(1):15, 2016.

Lehto, M. U. \& Järvinen, M. J. Muscle injuries, their healing process and treatment. Ann. Chir. Gynaecol., 80(2):102-8, 1991.

Majtan, J. Honey: an immunomodulator in wound healing. Wound Repair Regen. 22(2):187-92, 2014

Mitchell, C. A.; McGeachie, J. K. \& Grounds, M. D. Cellular differences in the regeneration of murine skeletal muscle: a quantitative histological study in SJL/ J and BALB/c mice. Cell Tissue Res., 269(1):159-66, 1992.

Molan, P. Re-introducing honey in the management of wounds and ulcers - theory and practice. Ostomy Wound Manage, 48(11):28-40, 2002.

Mori, R.; Kamei, N.; Okawa, S.; Nakabayashi, A.; Yokota, K.; Higashi, Y. \& Ochi, M Promotion of skeletal muscle repair in a rat skeletal muscle injury model by local injection of human adipose tissue-derived regenerative cells. J. Tissue Eng. Regen. Med., 9(10):1150-60, 2015.

Nakajima, Y.; Nakano, Y.; Fuwano, S.; Hayashi, N.; Hiratoko, Y.; Kinoshita, A.; Miyahara, M.; Mochizuki, T.; Nishino, K.; Tsuruhara, Y.; Yokokawa, Y.; Iuchi, T.; Kon, Y.; Mukai, K.; Kitayama, Y.; Murakado, N.; Okuwa, M. \& Nakatani, T. Effects of three types of japanese honey on full-thickness wound in mice. Evid. Based Complement. Alternat. Med., 2013:504537, 2013.

Pereira, T.; Gartner, A.; Amorim, I.; Armada-da-Silva, P.; Gomes, R.; Pereira, C.; França, L. M.; Morais, M. D.; Rodríguez, A. M.; Lopes, A. M.; Santos, D. J.; Luís, L. A. \& Mauricio, C. A. Biomaterials and stem cell therapies for injuries associated to skeletal muscular tissues. Adv. Biomaterials Sci. Biomed. Appl., 13:329-63, 2013.

Ranzato, E.; Martinotti, S. \& Burlando, B. Honey exposure stimulates wound repair of human dermal fibroblasts. Burns Trauma, 1(1):32-8, 2013.

Sánchez, D. L. \& Segovia, G. M. Análisis histológico de la aplicación de diferentes hidrogeles para la regeneración del músculo esquelético. Tesis de Grado en Medicina. Universidad de Valladolid. Facultad de Medicina. 2017. Disponible en: http://uvadoc.uva.es/handle/10324/24312.

Seckam, A. \& Cooper, R. Understanding how honey impacts on wounds: an update on recent research findings. Wounds Int., 4(1):20-4, 2013.

Schencke, C.; Sandoval, C.; Vásquez, B. \& del Sol. M. Quantitative analysis of dermal scars in deep skin burns treated with Ulmo honey supplemented with ascorbic acid. Int. J. Clin. Exp. Med., 11(3):2422-9, 2018.

Schencke, C.; Vásquez, B.; Sandoval, C. \& del Sol, M. El rol de la miel en los procesos morfofisiológicos de reparación de heridas. Int. J. Morphol., 34(1):38595, 2016.

Sherlock, O.; Dolan, A.; Athman, R.; Power, A.; Gethin, G.; Cowman, S. \& Humphreys, H. Comparison of the antimicrobial activity of Ulmo honey from Chile and Manuka honey against methicillin-resistant Staphylococcus aureus, Escherichia coli and Pseudomonas aeruginosa. BMC Complement. Altern. Med., 10:47, 2010.

Turner, N. J. \& Badylak, S. F. Regeneration of skeletal muscle. Cell Tissue Res., 347(3):759-74, 2012.

Vandamme, L.; Heyneman, A.; Hoeksema, H.; Verbelen, J. \& Monstrey, S. Honey in modern wound care: a systematic review. Burns, 39(8):1514-25, 2013.

Wolfensohn, S. \& Lloyd, M. Handbook of laboratory animal management and welfare. $4^{\text {th }}$ ed. Wiley-Blackwell, 2013.

\section{Dirección para correspondencia:}

Dra. Bélgica Vásquez

Universidad de Tarapacá

Arica- CHILE

Recibido : 16-10-2019

Aceptado: 25-11-2019

Email: bvasquezp@uta.cl 